NASA Technical Memorandum 83360

SAE-830729

\title{
New Test Techniques and Analytical Procedures for Understanding the Behavior of Advanced Propellers
}

George L. Stefko, Lawrence J. Bober, and Harvey E. Neumann

Lewis Research Center

Cleveland, Ohio

Prepared for the

1983 Business Aircraft Meeting and Exposition

sponsored by the Society of Automotive Engineers

Wichita, Kansas, April 12-15, 1983 
NEW TEST TECHNIQUES AND ANALYTICAL PROCEDURES FOR UNDERSTANDING THE BEHAVIOR OF AOVANCED PROPELLERS

\author{
George L. Stefko, Lawrence J. Bober, and Harvey E. Neumann \\ National Aeronautics and Space Administration \\ Lewis Research Center \\ Cleveland, Ohio 44135
}

\section{ABSTRACT}

New analytical procedures and experimental techniques are being developed to improve the capability to design advanced high speed propellers. Some results from the propeller lifting line and lifting surface aerodynamic analys is codes are compared with propeller force data, probe data and new laser velocimeter data. In general, the code comparisons with data indicate good qualitative agreement. A new rotating propeller force balance demonstrated good accuracy and reduced test time by 50 percent. Results from three new propeller flow visualization techniques are shown which illustrate some of the physical phenomena occurring on these propellers.

VERY HIGH PROPULSIVE efficiencies for aircraft that operate at subsonic speeds can be achieved with the free air propeller. This potentially high propulsive efficiency coupled with the concern to reduce direct operating cost through reduced fuel usage has led to a renewed interest in turboprop propulsion for high speed subsonic aircraft.

Both in-house and contractor studies indicated that there is a large performance advantage at cruise speeds up to Mach 0.8 for advanced high-speed turboprop powered a ircraft compared to equivalent technology high bypass ratio turbofans. These advantages could result in large block fuel savings, reduced life cycle costs, improved range, or other benefits for both future civil and military aircraft. To investigate these advantages, a high-speed Propeller Research Program was established at the NASA Lewis Research Center in 1976. Over the past six years, a number of advanced high-speed and low-speed propeller models have been tested. Results from these tests have shown that high propeller efficiencies can be obtained at cruise speeds up to at least Mach 0.8. Also, significant reductions in propeller noise have been demonstrated at cruise conditions.
This paper will present some of the latest propeller analytical procedures and test techniques used in the NASA Lewis Propeller Research Program. The latest propeller analytical procedures such as the curved lifting line and Euler lifting surface computer analyses will be briefly discussed and results compared with experimental data. Also, two new experimental tools, the laser velocimeter and the new rotating propeller force balance, will be reviewed. And finally, some propeller flow visualization techniques such as fluorescent mini-tufts, chemical sublimation and the paint flow technique will be discussed.

\section{ADVANCED TURBOPROP POTENT IAL}

The attractiveness of advanced turboprop propulsion results from its potential for very high propulsive efficiency at cruise speeds up to Mach 0.8. A comparison of the installed cruise efficiency of turboprop-powered and turbofan-powered propulsive systems is shown in Figure 1 over a range of cruise speeds. The efficiencies shown in the figure include the installation losses for both systems; namely, nacelle drag for the turboprop systems, and cowl drag and internal airflow losses for the turbofan systems. Conventional general aviation turboprops have installed efficiency levels near 80 percent up to about Mach 0.5 but can suffer from rapid decreases in efficiency above this speed due to increasing propeller compressibility losses. These losses are primarily the result of relatively thick blades ( 5 to 7 percent at 75 percent radius) used on many propulsion systems operating at high helical tip Mach numbers.

The advanced high-speed turboprop has the potential to delay these compressibility losses to a much higher cruise speed and achieve a relatively high performance to at least Mach 0.8 cruise. Although high bypass ratio turbofans exhibit their highest efficiency at cruise speeds near Mach 0.8 , their performance would 
still be significantly below that of the advanced turboprops. At Mach 0.8 the installed efficiency of turbofan systems would be approximate 7 y 65 percent compared to about 75 percent for the advanced turboprop. At lower cruise speeds, the efficiency advantage of the advanced turboprop would be even larger.

A number of studies have been conducted by both NASA and industry to evaluate the potential of advanced high-speed turboprop propulsion for both civil and military applications. Numerous references to specific studies and summary results are listed in Reference 1. The trip fuel savings shown in Figure 2 as a function of operating range is a summary of these studies. Installed efficiency levels similar to those shown in Figure 1 for comparable technology advanced turboprops and turbofans were used in most of these studies. As shown in Figure 2, trip fuel savings is dependent on aircraft cruise speed and range. At the bottom of the band, associated with Mach 0.8 cruise, fuel savings range from about 15 to 25 percent for advanced turboprop aircraft compared to equivalent technology turbofan aircraft. The larger fuel savings occur at the shorter operating ranges where the mission is climb and descent dominated. Because of the lower operating speeds encountered during climb and descent, turboprops have an even larger performance advantage than the advantage at Mach 0.8 cruise conditions. In a similar manner, a larger fuel savings is possible at Mach 0.7 cruise (represented by the top of the band in Fig. 2). At this lower cruise speed, fuel savings range from about 25 to near 35 percent. Even larger fuel savings may be possible by recovering the propeller swirl loss from these single rotation turboprops. Counterrotation is one promising concept for recovering swirl loss that is currently under study at NASA and in the industry.

ADVANCED DESIGN CONCEPTS AND ANALYTICAL PROCEOURES

To achieve these possible fuel savings, the propeller on the advanced turboprop would have to incorporate a number of unique design features that would enhance propeller performance and lower source noise. For example, a propeller designed for a cruise Mach number of 0.80 at an altitude above $9.144 \mathrm{~km}(30,000 \mathrm{ft}$.$) ,$ would have local blade Mach numbers from just over 0.8 at the blade hub to supersonic Mach numbers (near 1.15) at the blade tip. Thus, the concepts shown in $\mathrm{Figure} 3$ are required to reduce blade compressibility losses and attain high efficiency. These concepts include proper shaping of the nacelle to reduce inboard blade Mach number, blade sweep to reduce outboard blade local Mach number, thinner blades to reduce drag rise Mach number and spinner area ruling to prevent in-board blade choking. In addition, to hold propeller diameter to a reasonable value, a high power (or disk) loading and concomitantly a large number of blades
( 8 or 10) are required. The inboard portion of the propeller then operates as a cascade rather than isolated blades.

The above design concepts along with the attendant mixed subsonic and supersonic flows present a complicated propeller design problem that is beyond the scope of traditional propeller analytical procedures. Therefore, advanced aerodynamic analysis methods for predicting high-speed propeller performance are being developed as a part of the NASA Propeller Research Program (2-4). The analys is methods (Fig. 4) range from simple short running lifting 1 ine programs such as the existing strip analys is based upon Goldstein's work (5) to very complex long running programs such as the finite difference lifting surface analysis that solves the five Euler equations $(3,6)$.

The existing Goldstein type strip analysis assumes the vortex wake is composed of a rigid helical vortex sheet, corresponding to the optimum span-wise loading of a lightly loaded propeller. The propeller is restricted to having straight blades and there is no provision for a nacelle since the vortex wake extends to the axis. Simple modifications have been made to existing analyses in an attempt to circumvent these restrictions. For example, the simple cosine rule is used to account for blade sweep and a radial gradient of axial velocity is used to account for the effect of the nacelle.

More recent lifting line analyses, such as the curved lifting line program (7) and the propeller nacelle interaction program (8) include a swept lifting line capability, and to varying degrees, the ability to account for the presence of the nacelle in the analysis.

The curved lifting line analys is represents the wake by a finite number of helical vortex filaments instead of the continuous sheet of vorticity used by Goldstein. Each filament has a constant pitch but its location relative to the other filaments is arbitrary. The strengths of the individual wake filaments are related to the spanwise variations of the bound vortex strength. Since both of these are unknown, the blade and wake vortex strengths are solved simultaneously. This solution is made possible by placing the bound vortex along the quarter chord $l$ ine and requiring the flow to be tangent to the blade mean camber line along the threequarter chord line. With these conditions, the blade and wake vortex strengths can be computed. The lift coefficient of the blade at any radial location is then determined from the bound vortex strength at the same radius. Drag is provided by correlated two-dimensional airfoil data. The total induced velocity at any point in the flow field is obtained by summing the induced flow of the bound vortex and the trailing vortex system.

This analysis is currently restricted to single rotation propellers. The blades are represented by curved lifting lines which can have any arbitrary shape. The nacelle shape is restricted to an infinite cyl inder because the 
wake rigid helical filaments cannot contract in the radial direction. The radially varying propeller inflow velocity due to nacelle curvature can be prescribed and taken into account in the analysis.

The propeller nacelle interaction analys is (8) also represents the wake by a finite number of vortex filaments. However, this analys is allows the vortex filaments to be placed along stream surfaces so that they conform to the shape of an axisymmetric nacelle. This is accomplished in the analys is by the calculation of the inviscid flow around the nacelle alone, which locates the wake vortex filaments around the nacelle and determines the radial variation of the inflow velocity at the propeller. The induced velocity is determined by summing the induced flow from the individual filaments and the swept lifting line. The local blade lift and $d r a g$ are determined from two-dimensional airfoil and cascade data contained in the progranl and the calculated local blade angle of attack. An optional step in the analys is allows the calculated blade forces to be used in a circunterentially averaged (axisymmetric) viscous compressible flow calculation to determine interblade and off-body velocities. This calculation can indicate whether the velocities are high enough to result in large shock wave losses, and can determine the drag of the nacelle in the presence of the propeller. The wake model in this analys is can be applied to both single and coaxial counter-rotation propellers. The propellers can have blades of any arbitrary shape and the nacelle can be any axisymmetric geometry.

Lifting surface analyses that can solve the complete three-dimensional flow field are also under development. These analyses require the generation of a complex grid system which conforms to the shape of the nacelle and propeller. The nacelle shape is required to be axisymmetric so that the flow between each two adjacent blades is the same, and it is only necessary to solve for the flow between two blades. Beyond the tips and upstream and downstream of the blades, the flow is assumed to be periodic. On all solid surfaces the flow is required to be tangent to the surface.

One lifting surface analys is that is under development solves the single full transonic potential equation (4). It requires the wake location to be defined and does not account for shock total pressure variation al though it indicates the shock location. Another lifting surface technique is the Euler analys is $(3,6)$. The equations used in the Euler analys is are the five unsteady three-dimensional Euler equations. These govern the inviscid flow of a compressible fluid and can accurately represent the total pressure variation caused by shock waves and the work done by the propeller. This analysis requires no wake modeling and no twodimensional airfoil data. This code has a significantly longer running time than the transonic potential analysis. Both of the lifting surface analyses will be able to predict de- tailed pressure distributions on both sides of the propeller blade as well as the flow conditions in any portion of the off-body flow field. Such detailed three-dimensional results will be important tools for improving the aerodynamic, acoustic and structural performance of propellers through a better understanding of the complicated flow processes of advanced high-speed propellers.

\section{EXPERIMENTAL DATA COMPARISONS WITH ANALYTICAL PROCEDURES}

Although these lifting line and lifting surface programs are still under development, some comparisons of their results with wind tunnel experimental data obtained with a laser velocimeter $(L V)$, a rotating force/torque balance and a flow survey probe on an 8-bladed, $45^{\circ}$ swept propeller (SR-3) will be made herein (see also Ref. 9). These comparisons are intended to indicate the current status in the development of the programs.

The first analytical/experimental data comparisons will be made with wind tunnel experimental data obtained using the new laser velocimeter (LV) system. The laser velocimeter system was developed to obtain non-intrusive measurements of detailed velocities ahead of, in between, and behind propeller blades (10-12). The laser system is shown in Figure 5 instalied in the NASA Lewis 8- by 6 -foot wind tunne?. The SR-3 propeller, spinner, and nacelle can also be seen in this figure.

This laser velocimeter system uses a 15-watt argon ion laser which is operated at about $1 / 3$ power. The system uses a four beam on-axis backscatter optic system. The measuring volume is moved axially and vertically within the wind tunnel by traversing the entire laser system and is moved horizontally by using a zoom lens assembly. The movement of the measuring volume is remotely computer controlled. The flow with in the tunnel is artificially seeded with dioctyl phthalate (DOP). The four beam laser velocimeter is capable of measuring two velocity components simultaneously. The four beams were set up such that the planes defined by the two beams of each color were essentially orthogonal to each other and at nominally $45^{\circ}$ to the horizontal plane of the wind tunnel. The axial and tangential components of velocity were obtained by making a measurement in the horizontal plane which passes through the propeller rotationa? axis. The axial and radial components of velocity were similarly obtained by making measurements in the vertical plane which passes through the rotational axis.

In Figures 6 and 7 , the laser velocimeter velocity data is compared with results from the curved 1 ifting line analys is. The comparisons shown are for the 8-bladed, $45^{\circ}$ swept (SR-3) propeller (shown in Fig. 5) operating at a tunnel Mach number of 0.8 , a design advance ratio of 3.06 , a corrected tip rotational speed $\left(v_{t} / \sqrt{\theta}\right)$ of $280 \mathrm{~m} / \mathrm{sec}(917 \mathrm{ft} / \mathrm{sec})$, a helical tip Mach number of 1.15, a power coefficient of 
1.8 , and a blade angle setting at the threefourths radius of $60.5^{\circ}$.

A color computer graphic technique similar to References 13 and 14 has been utilized to evaluate and compare the measurements and predictions of the highly three-dimensional exit flow from the propeller. At an axial station just behind the propeller, laser velocimeter (LV) circumferential surveys were taken at 17 radial positions located from 59 to 122 percent of the blade span. These data were used to generate the computer graphic presentations of Figure 6. At each radial position, the data over the complete circumference for the 8 blades was folded to a $45^{\circ}$ segment to provide data in a single equivalent blade passage. The circumferential data was averaged to provide $30 \mathrm{cir}-$ cumferential values with in this "equivalent" blade passage. The entire array of experimental data at 17 radial positions by 30 circumferential positions was interpolated to provide data at intermediate positions, color-coded and displayed on a color raster display. The results were photographed to provide the results shown in the figure. The analytical results were processed in an identical manner. Velocities were predicted for the same 17 by 30 array of spatial positions, computer interpolated and graphicaliy displayed.

Figure 6 presents the axial velocity field in the absolute reference frame seen by a stationary observer. The blade rotation in the figure is in the counterclockwise direction when viewed from behind the propeller looking forward. The axial velocity measured by the laser velocimeter clearly show the thick blade wakes and the tip vortex. The light gray region adjacent to the blade wakes is the high velocity from the suction surface. The maximum suction surface velocity occurs at about 0.9 of the tip radius. In making the comparison of the LV data with the results from the curved lifting line analysis, the viscous blade wakes must be ignored since they are not included in the analysis. The comparison also shows the analys is has a stronger predicted tip vortex because of the mathematical singularity present in the analys is at the tip. The general character of the flow field as measured is strikingly similar to the flow field predicted by the curved lifting line analysis. Some flow disturbances are noted beyond the blade tip. These velocity fluctuations may present a potential noise source. In general, the curved lifting line analys is shows good qualitative agreement with the experimentally measured LV data.

In Figure 7 , the resultant velocity fields (i.e., the vector sum of the axial and tangential velocities) between the blades at a radius ratio of 0.79 are shown for the LV data and the curved lifting line analytical results. The flow direction is from left to right and the blade rotation from top to bottom. A short portion of the flow ahead of the blade is shown in addition to the flow within the blades and slightly downstream of the blades. The data shown is for axial distances of $x / R=0.32$ to $x / R=0.94$. The blade extends from $x / R=0.53$ to $x / R=0.82$. The results have been folded to an included angular displacement of $45^{\circ}$. For presentation purposes, the folded results have been repeated twice in the figure to show two blade passages.

The LV picture shows a rapid acceleration on the suction side of the blade. Other numeric LV data not presented indicate a shock wave just downstream of the high velocity region. The data indicate that the shock, however, does not extend completely across the blade passage. The curved lifting line analys is does not include provision for shocks although the analys is does indicate correctly the rapid acceleration on the suction surface. The LV data indicates mild accelerations upstream of the blades. This effect also appears in the analysis; however, the velocity gradients appear to be larger in the analysis. The larger gradients in the analys is results are due to the concentration of the load at the quarter chord of the blade, whereas in reality, the load is distributed along the chord. The general character of the velocity field, however, show qualitative agreement between the curved lifting line predictions and the measured LV results.

The SR-3 propeller test results obtained with the original rotating balance installed in the propeller test rig and a flow survey probe are compared in Figure 8 with the curved lifting line and the propeller/nacelle lifting line analytical codes. For consistency of comparison with experimental results, the analytical results account for the change in blade twist resulting from centrifugal forces generated by blade rotation. These data are presented over a range of advance ratios for a free-stream Mach number of 0.8 . In the power coefficient curve, both analytical methods overpredict the power coefficient although the curved lifting Iine analysis more accurately predicts the level. Both methods deviate further from the data at both high and low advance ratios and are most accurate in the mid-range. The assumed variation of twist change with rotational speed affects the shape of the power coefficient curve. The assumed variation of twist with rotational speed squared may be responsible for some of the discrepancy in the predicted and measured power coefficient results. For the efficiency curves, the curved lifting line analys is adequately agrees with the data while the propeller nacelle interaction analys is considerably underpredicts the efficiency at low advance ratios and considerably overpredicts the efficiency at high advance ratios. The differences between the results from the two methods appear to be primarily due to the different approaches used for obtaining lift.

Comparisons for radial distribution of loading are shown in Figure 8 (b) for the $S R-3$ propelier at a free-stream Mach number of 0.8 and an advance ratio of 3.06 . The propeller nacelle interaction analys is accurately predicts the loading distribution over most of the blade, deviating appreciably only in the outer 20 per- 
cent of the blade. The curved lifting line analys is overpredicts the loading inboard and underpredicts outboard, both by appreciable amounts. Since the experimental radial loading distribution was obtained from a probe located downstream of the propeller, any blade wake rollup would cause the measured loading distribution in the wake to be different from the actual loading distribution on the blade. Also, changes in the flow streamlines from the propeller to the probe, caused by changes in the nacelle diameter, would cause the loading distributions to be somewhat different at the two axial locations. Because of these uncertainties in the data, it is not possible to conclude which method more accurately predicts the loading distribution.

A comparison of the analytical and experimental results recently obtained with the Euler lifting surface analys is can be seen in Figures 9 through 13.

In Figure 9, the predicted relative Mach number on the surface of the SR-3 propeller blades and between the blades are shown for the SR-3 propeller at a free stream Mach number of 0.8 , an advance ratio of 3.06 , and a blade angle of 61.3 degrees. The level of Mach numbers on the suction side of the blade near the nacelle surface at about the two-thirds chord indicates the presence of a shock wave at that location. On the pressure side of the blade, a shock wave is also indicated at about the same location. The middle portion of the figure shows the calculated Mach numbers between the blade passages near the hub and at 82 percent of the blade span. Near the hub, the shock wave is strong and spans the entire passage. At the 82 percent blade span location, the shock wave eminates from the suction surface near the trailing edge but becomes very weak near the pressure surface.

The results shown in Figure 9 are in good qualitative agreement with laser velocimeter measurements presented in Reference 12. One significant difference is the location of the shock wave. In the computed results, the shock wave originates closer to the trailing edge of the blade on the suction surface whereas the data of Reference 12 indicates a shock wave location somewhat upstream of the trailing edge. This behavior is consistent with shock wave boundary layer interactions in other types of flow fields in which viscous effects cause the actual shock wave location to be upstream of the location predicted by inviscid analyses.

The Euler analys is results are next compared in Figures 10-13 with experimentally measured propeller wake swirl angles and power coefficients at the free stream Mach numbers of 0.6 and 0.8 . In Figure 10, for a free stream Mach number of 0.6 , the Euler analys is values of swirl angle downstream of the propeller are compared with experimentally measured values of swirl angle obtained during the wind tunnel tests described in Reference 15. These values were measured with an instrumented wedge mounted on a translating probe. Both the computed and measured values correspond to an axial location
0.21 propeller diameters downstream of the pitch change axis. Although the level of the predicted results is considerably higher than the experimental results, the radial variation of swirl is in reasonable agreement between the two sets of results when an estimate of viscous effects is included in the comparison using the compressor methodology of Reference 16. At a radius ratio $r / R=0.52$ which corresponds to a solidity (chord/gap) of 1.0 , the deviation angle was determined to be 3.15 degrees. This corresponds to a reduction of the swirl angle to a value of 5.8 degrees from the originally predicted 8.9 degrees. The unusual behavior of the data near $r / R=1.0$ is apparently caused by the tip vortex rollup. The analys is has not predicted this feature of the flow because the mesh is too coarse in this region.

A comparison of the Euler computed and measured propeller power coefficient is shown in Figure 11. Experimental results are taken from Reference 15. The power coeficient is considerably overpredicted. Since power coefficient is closely related to swirl angle, the overprediction of power coefficient is consistent with the overprediction of swirl angle shown in Figure 10. It appears that at lower free stream Mach numbers, the overprediction of power coefficient at this Mach number is due to neglecting viscous effects in the analys is and that boundary layer growth on the blade surface causes a reduction in swirl angle downstream of the propeller.

Predicted and measured swirl angle downstream of the propeller at a free stream Mach number of 0.8 are shown in Figure 12. Numerical results for swirl angle are higher than those measured in the wind tunnel (15) and are similar to the results at a Mach number of 0.6 ( $\mathrm{Fig} .10$ ). The swirl angle overprediction is approximately equal to four degrees whereas at a free stream Mach number of 0.6 the discrepancy is about three degrees. This difference implies that at free stream Mach number of 0.8 , a mechanism in addition to boundary layer growth is causing decreased flow turning. This mechanism could be due to the presence of the previouslydiscussed shock wave located just upstream of the blade trailing edge. A shock wave boundary layer interaction resulting from this shock location would result in reduced blade loading and cause reduced swirl relative to a trailing edge shock wave location. This fact would at least partially explain the larger overprediction of swirl angle shown in Figure 12. The compressor methodology of Reference 16 cannot be used to estimate viscous losses at this condition. This methodology is based on low speed cascade data and the presence of shock waves in the flow field clearly makes it not applicable. The radial variation of swirl angle agrees reasonably well with the experimental results. The region with the largest discrepancy is again the tip region where the coarse mesh cannot resolve the details of the flow associated with the tip vortex. 
A summary of propeller power coefficient for the SR-3 propeller at free stream Mach number of 0.8 is shown in Figure 13 . The SR-3 propeller was designed for a cruise condition at a free stream Mach number of 0.8 , advance ratio of 3.06 and a power coefficient of 1.7 . The power coefficient is shown for three blade angle settings. Although the power coefficient is again overpredicted, the variation of power coefficient with blade angle is predicted quite well. The overprediction of power coefficient is consistent with the overprediction of swirl angle shown in Figure 12 and would improve if shock wave boundary layer interaction and $v$ iscous effects were included in the analys is.

\section{NEW ROTATING PROPELLER BALANCE}

Accurate propeller performance testing is a difficult task which requires special test equipment. As an example, fan performance for a turbofan engine can be measured accurately using conventional flow survey techniques because of the large 50 to 70 percent pressure rise across it. Advanced propellers, however, have only about a 5 percent pressure rise, so sophisticated force balances are required to accurately measure the performance. This section summarizes the development of an accurate rotating force balance for measurement of both thrust and torque on propeller models.

The rotating balance is located in the NASA Lew is $746 \mathrm{KW}$ (1000 hp) Propeller Test Rig (PTR) as shown in Figure 14. This PTR can be installed in the Lewis $8-$ by $6-, 10-$ by $10-$, or 9- by 15 -foot wind tunnels to investigate a range of test speeds from Mach 0 to 0.85 . Power for this test rig is provided by an air turbine which is $15 \mathrm{~cm}$ ( $5.9 \mathrm{in}$ ) in diameter and a continuous flow air system supplying $3.1 \times 10^{6}$ $\mathrm{N} / \mathrm{M}^{2}$ (450 psi) air which is routed through the support strut.

The original rotating force balance had thermal drift and rotational interaction problems which required special test procedures and extra tests to obtain accurate data. Because of these problems, it was necessary to conduct two different wind tunnel tests to measure propeller performance. An initial test was conducted using the strut load cell (Fig. 14) to establish a windmill drag level. Then a second test was conducted to measure the incremental thrust from windmill to a powered condition and back to windmill. The original rotating balance was used to obtain this incremental thrust over a very short period of time where thermal drift errors would be insignificant. This special test procedure is described in more detail in Reference 15. To eliminate the long test times and the problems with the original rotating balance, a new rotating balance was designed and built through a Lewis contracted program with Shaker Research Corporation. The calibration and initial wind tunnel testing was performed by NASA Lewis personnel in the Lewis 10 - by 10-foot wind tunnel at speeds to Mach 0.35 .
Some of the key features in the new rotating balance can be seen in the cross-sectional view of the balance in Figure 15. The dark sections in the figure represents the rotating part of the balance. To minimize any thermal drift problems with the new balance, it was designed with water cooling passages to carry away the heat generated by the bearings. In addition, the main heat generating thrust bearing was located at the rear of the balance in order to be as far away as possible from the thrust and torque sensors which were located at the front of the balance. To minimize any rotational interaction problems, the thrust and torque strain gauges were mounted on two rows of four radial spokes. Two front and two rear spokes can be seen in this figure. A photograph of the thrust and torque radial spokes can be seen in Figure 16 .

Extensive static and spin calibrations were performed in the 10- by 10-foot wind tunnel to determine the balance constants and to understand the behavior of the new balance. A photograph of the static torque calibration rig is shown in Figure 17 .

Some typical propeller test data acquired with the new rotating balance is presented in Figure 18. This data shows the propeller power coefficient and net efficiency versus advance ratio at the takeoff Mach number of 0.2 for the SR-2, 8-bladed, unswept propeller (17). The square symbols signify data acquired with the original balance system while the circles represent data acquired with the new rotating balance. Near the takeoff design condition $\left(C_{p}=1.0, J=0.875\right)$, both balances measure essentially the same power coefficient. With the new rotating balance, a slightly lower net efficiency of 50.5 was obtained compared to 51 percent for the original balance systern at this design advance ratio. Similar favorable agreement existed up to an advance ratio near 1.5 . At higher advance ratios above 1.5, however, a discrepancy in net efficiency exists between the two force measurement systems. This discrepancy near peak propeller efficiency is in the region where very small forces are measured and propellers do not normally operate during low speed take-off and climb. Also, the peak efficiency of 91.3 percent measured with the original balance system appears to be too $\mathrm{high}$ when compared to analytical predictions. In general, the good agreement between the two balance systems over the operating range of interest gives credibility to the new rotating balance since the original force measurement system had (in Ref. 15. Appendix A) previously been shown to be in good agreement with data taken with another propeller test $r i g$ in the United Technologies Research Center (UTRC) wind tunne1.

In summary, wind tunnel tests on the SR-2 two-foot propeller model at take-off and $\mathrm{climb}$ condition have demonstrated that the new propeller rotating balance accurately measured the propeller's thrust and torque. Also, the new balance cut the propeller test time in about 
half because it eliminated the need for two separate wind tunnel tests as was required with the original force measurement system. This large savings in test time should result in significant cost savings to NASA for all future propeller tests.

\section{FLUORESCENT MINI-TUFT TECHN IQUE}

The fluorescent mini-tuft technique is a flow visualization method that can be used to understand the surface flow field on operating propeller blades and locate potential stalled regions. This technique (18-20) is an extension of the classic tuft flow visualization method to propeller testing.

In the fluorescent mini-tuft technique, very tiny (e.g., diameters of 0.0005 to 0.002 inch) nylon-tufts are glued to the propeller blade surface. The tufts are made visible by using a special high power fluorescent stroboscope. The tuft motion is then recorded on video tape and $35 \mathrm{~mm}$ photographs using a special photographic system.

The extremely small tufts are used to minimize the centrifugal forces acting on the tufts so that the aerodynamic forces can have more influence on the tufts. The reason that the centrifugal force diminishes faster than aerodynamic force as the tuft diameter is decreased is that the centrifugal force depends on the mass of the tuft which is proportional to the tuft cross-sectional area or diameter squared. on the other hand, the aerodynamic force is proportional to the tuft projected frontal area (i.e., tuft diameter times the tuft length perpendicular to the flow). Thus a smaller tuft diameter causes centrifugal forces to diminish faster than aerodynamic forces and thus allows the tuft to respond more to aerodynamic forces. The small tuft diameter also minimizes the aerodynamic drag which helps to make this method a more non-intrusive flow visualization method.

The tiny size of the tufts, however, creates the problem of seeing and recording them. This problem is overcome by making the tufts appear larger than they actually are by using fluorescent photography. A special ultraviolet stroboscope fires a burst of ultraviolet energy in one millionth of a second. The ultraviolet energy causes the tufts to fluoresce while the rapid pulse stops the motion of the propeller which may be rotating at speeds up to 9000 RPM in the propeller test rig. The fluorescing tufts emit visible light which makes the tufts appear 10 to 20 times larger than they actually are. A $35 \mathrm{~mm}$ camera with a filter that only allows visible light to pass into the camera, records the image of the enlarged tufts.

In conjunction with the above equipment, an electronic angle encoder along with a video camera and stroboscope is used to position the propeller blade to any desired position with 0.1 degrees so that the desired $35 \mathrm{~mm}$ picture is obtained. Further details of the electronic equipment can be found in Reference 21 .
A fluorescent mini-tuft picture of the SR-6, 10-bladed, 40 cegree swept propeller is shown in Figure 19. The propeller is in the NASA Lewis 10- by 10-foot wind tunnel operating at a condition near climb (i.e., Mach number of 0.35 , rotational speed of 5100 RPM, and a blade angle of 57 degrees). The fluorescent mini-tufts indicate that the local blade flow is well behaved and that there is no significant separated flow regions at this condition.

Figure 19 illustrates the type of flow visualization results that can be achieved on rotating propeller blades. The method may also have application to other rotating hardware such as helicopter blades or turbomachinery. The technique can survey the flow field while other tests are being conducted with minimal interference to the flow.

\section{PROPELLER BOUNDARY LAYER TRANS ITION LOCATION USING SUBL IMATING CHEMICALS}

In wind tunnel testing, incorrect boundary layer transition locations can result in unrealistic aerodynamic predictions when the wind tunnel data is applied to a flight vehicle. Therefore, a sublimating chemical flow visualization technique that has been used successfully with fixed wing aircraft (22) was applied to these SR-6 propeller models to determine where the boundary layer transitions from laminar to turbulent flow.

The SR-6, 10-bladed, 40 degree swept propeller was tested in the NASA Lewis 10 - by 10-foot wind tunnel to develop this propeller sublimation technique. In this test, the surface under observation is coated with a thin film of a volatile chemical solid (fluorene in a solvent). The technician in Figure 20 is shown applying the chemical with a paint sprayer while wearing a Scott Air Pack to avoid breathing the potentially toxic fumes. When the air flows over the model, the chemical sublimes in proportion to the local shear stress, heat transfer and vapor pressure of the sublimating chemical. Thus, when the boundary layer transitions from laminar to turbulent flow with its attendent $h i g h e r$ shear stress and mixing, the chemical will sublime from the surface where a turbulent boundary layer exists but still remain on the surface where the laminar boundary layer exists. The resulting demarcation line tells where the boundary layer transitions.

The chemical coating consisted primarily of fluorene. The fluorene was mixed with the solvent 1.1.1 trichloroethane in a one to eight ratio by volume in order to apply it with a paint spray gun in the desired pattern. The pattern for propellers requires a radial varying chemical thickness in proportion to the local blade velocity so that a uniform chemical coating remains at the end of the test. For the SR-6 propeller operating at a climb test condition, the helical Mach number over the blade ranged from about Mach 0.35 near the hub to Mach 0.84 at the tip. Corresponding to these 
Mach numbers, a chemical coating around 0.001 inches was applied near the hub and about a 0.002 inch coating was applied near the tip.

A stroboscopic picture of the SR-6, 10bladed, 40 degree swept propeller during a chemical sublimation test in the Lewis 10- by 10-foot wind tunnel is shown in Figure 21. The propeller is operating at a climb condition with a rotational speed of 7340 RPM, a Mach number of 0.35 , an advance ratio of 1.41 , a power coefficient of 1.59 , and a blade angle of $B 3 / 4=49^{\circ}$. The photograph was taken near the end of the test after the tunnel had been running for about 15 minutes. The chemical remaining on the top two blades indicate that the boundary layer transitions from laminar to turbulent flow at about 15 percent of the propeller blade chord. This transition location is similar to what would be expected in flight on a full scale propeller with a 10 to 15 foot diameter. Thus, this chemical subiimation technique is another useful experimental tool for understanding propeller boundary layer flows.

\section{PROPELLER "PA INT FLOW" TECHN IQUE}

A performance comparison of a comparable 8and 10-bladed propeller (23) is shown in Figure 22 where net efficiency is plotted versus Mach number. These data show both propellers operating at the 10-bladed propeller design power coefficient and advance ratio. The performance of the 10-bladed propeller was about $3 / 4$ to 1 percent higher than that of the 8-bladed model from Mach 0.6 to about 0.76 . This higher performance would be expected due to the higher ideal efficiency for a blade count increase from 8 to 10 . For the 8-bladed propeller, the performance loss due to compressibility effects began above Mach 0.7 and increased gradually with increasing speed. The 10-bladed propeller, however, showed no performance loss up to a speed near Mach 0.75. Beyond this speed, the efficiency fell rapidly with increasing Mach number. By Mach 0.8 , the efficiency had fallen 3 percent and was $1 / 2$ percent below the 8 -bladed model. To better understand this large 3 percent efficiency loss, a new flow visualization technique, called the paint flow technique, was developed.

The paint flow technique consists of painting the propeller blades with a red undercoat and a white overcoat. Then with the propeller operating at the desired test condition, an upstream jet atomizer was turned on to produce a cloud of dioctyl-phthalate (DOP) particles. The DOP solvent then impinges on the rotating propeller blades and after about 30 minutes of testing causes the paint to flow, etching the surface airflow patterns into the surface of the blades.

The results of these paint flow tests with the 10-bladed propeller operating near design at Mach 0.8 is shown in Figure 23. The photographs indicate a rather extensive shock on both the pressure and suction sides of the pro- peller blade. The shock structure extends far from the hub region to near the blade midradius. The extensive shock structure indicates that hub choking was quite severe, and propagated outward over a considerable portion of the blade span. Thus, these photographs provide convincing evidence that hub choking was responsible for most of the 3 percent loss in efficiency shown in Figure 22.

The new computer analyses previously described are now being used to design propellers that will minimize this hub choking problem. Thus, this paint flow technique represents another useful experimental tool to improve the understanding of the physical phenomena occurring on these advanced propellers.

\section{CONCLUDING REMARKS}

Some of the latest propeller analytical procedures and experimental techniques used in the NASA Lewis Propeller Research Program have been presented.

The analytical curved lifting line code was compared with data from a new experimental tool, the laser velocimeter. In general, the data comparisons indicated good qualitative agreement. Also, prediction from two lifting line codes were compared to propeller force and flow survey probe data. These comparisons showed reasonable agreement in terms of propeller efficiency and power coefficient but only fair characterization of the propeller radial loading distribution. Thus, these new lifting line codes are useful as preliminary design methods to determine which of the potential propeller designs should go on to a more detailed analys is with the lifting surface analytical codes.

The Euler lifting surface code was briefly reviewed and results were presented which showed it has the capability of predicting shock waves and the detailed inviscid features of the flow. The predicted propeller swirl angles and power coefficients were overpredicted because the analysis does not include viscous effects at this time. However, the radial variation of swirl angle downstream of the propeller was predicted reasonably well. Also, the variation of propeller power coefficient with blade angle was predicted very well. Analys is work is planned to add viscous effects to this Euler method. With viscous effects added to the analysis, the Euler propeller analys is code should represent a significant advance in propeller design methodology.

A new rotating thrust and torque balance for model propeller tests was recently calibrated and evaluated in the Lewis 10 - by 10 -foot wind tunnel. This new balance reduces propeller wind tunnel test time by one half and can accurately measure performance of two foot propeller models as compared to the original propeller force measurement system that was used at Lewis. This new rotating balance significantly simplifies and speeds up propeller wind tunnel testing with the potential of large cost savings in future model tests. 
Although this new propeller balance provides excellent quantitative data about the propeller's performance (e.g., thrust and torque), it cannot provide information on the detailed propeller flowfield. For this information, several flow visualization methods such as the fluorescent mini-tuft technique, the chemical sublimation method, and the paint flow technique were developed. Each of these techniques adds a special capability to obtain qualitative flowfield information which show the physical phenomena occurring on these advanced propellers. For example, the fluorescent mini-tuft technique can show any separated regions on the propeller. The chemical sublimation method identifies where the boundary layer transitions from laminar to turbulent flow which can help verify that wind tunnel data can be aerodynamically scaled to flight conditions. And the paint flow technique shows the surface flow pattern which, if an abrupt change in flow conditions exists, could show the location of these changes such as shock waves. Thus, all of these flow visualization techniques have accomplished a specific goal and have provided a better understanding of the physical phenomena occurring with these advanced propellers.

In summary, the experimental and flow visualization methods contribute to the improvement and verification of the new advanced analytical methods that are under development. These analytical methods, in turn, should give greatly improved capability for designing future ad$v$ anced propellers with enhanced performance characteristics.

SYMBOLS

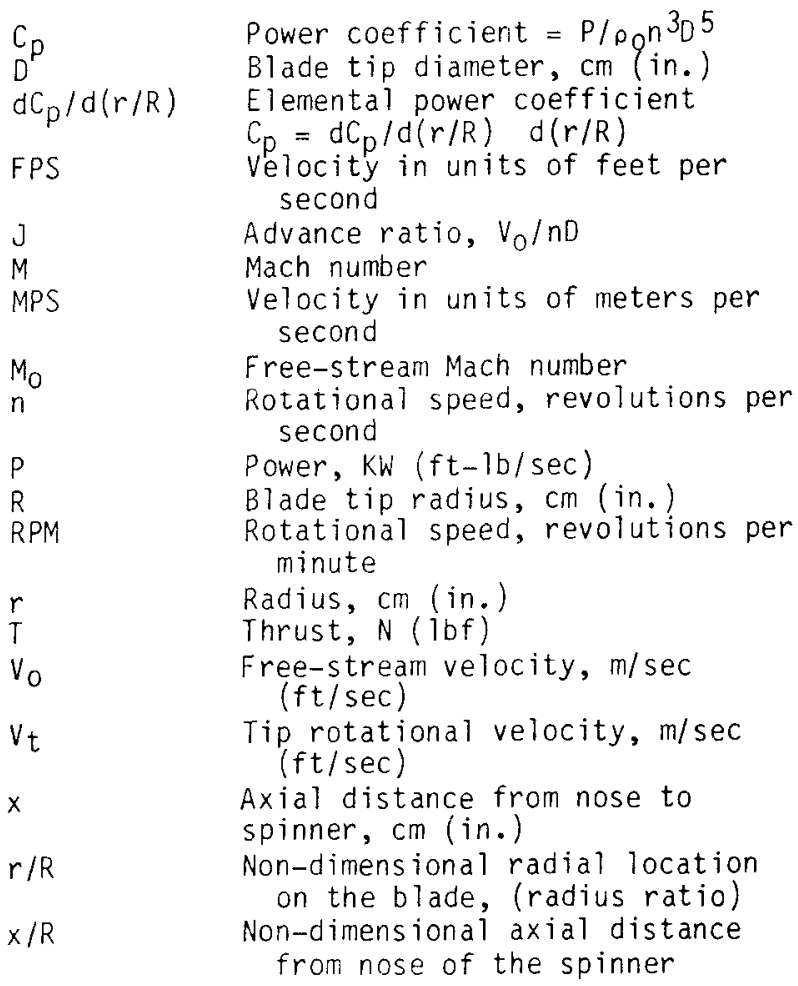

Propeller blade angle at three-quarter radius Efficiency $=\left(T \cdot V_{0}\right)$ P

Ratio of total temperature to standard sea-level temperature of $518.7^{\circ} \mathrm{R}$

р0

Free-stream density, $\mathrm{kg} / \mathrm{m}^{3}$ (slugs $/ \mathrm{ft}^{3}$ )

\section{REFERENCES}

1. R. J. Jeracki, D. C. Mikkelson and B. J. $B l$ aha, "Wind Tunnel Performance of Four Eriergy Efficient Propeliers Designed for Mach 0.8 Cruise," SAE Paper 790573, April 1979 (NASA TM-79124).

2. L. J. Bober and G. A. Mitchell, "Summary of Advanced Methods for Predicting High Speed Propeller Performance," A IAA Paper 80-0225, January 1980 .

3. L. J. Bober, D. S. Chaussee and P. Kutler, "Prediction of High Speed Propeller Flow Fields Using a Three-Dimensional Euler Analysis," A IAA Paper 83-0188, January 1983.

4. W. H. Jou, "Finite Volume Calculations of Three-Dimensional Flow Around a Propeller," A IAA Paper 82-0957, June 1982.

5. S. Goldstein, "On the Vortex Theory of Screw Propellers," Royal Society (London) Proceedings, Vol. 123, No. 792, April 6, 1929, pp. 440-465.

6. D. S. Chaussee and P. Kutler, "User's Manual for Three-Dimensional Analysis of Propeller Flow Fields, "NASA CR-167959, January 1983.

7. J. P. Sullivan, "The Effect of Blade Sweep on Propeller Performance," A IAA Paper 77-716, June 1977 .

8. T. A. Egolf, 0. L. Anderson, D. E. Edwards and A. J. Landgrebe, "An Analys is for High Speed Propeller-Nacelle Aerodynamic Performance Prediction; Volume 1, Theory and Initial Application and Volume 2, User's Manual for the Computer Program," United Technologies Research Center, R79-912949-19, June 1979.

9. L. T. Bober and L. K. Chang, "Factors Influencing the Predicted Performance of Advanced Propeller Designs," NASA TM-82676, 1981 .

10. J. S. Serafini, J. R. Sullivan and H. E. Neumann, "Laser-Velocimeter Flow-Field Measurements of an Advanced Turboprop," A IAA Paper 81-1568, July 1981.

11. R. J. Freedman and J. P. Greissing, "The Development and Utilization of a Laser Velocimeter System for a Large Transonic Wind Tunnel, "NASA TM-82886, 1982 
12. H. E. Neumann, L. J. Bober, J. S. Serafini and L. K. Chang, "An Analytical and Experimental Comparison of the Flow Field of an Advanced Swept Turboprop," A IAA Paper No. 83-0189, January 1983.

13. B. H. Anderson, C. W. Putt and C. C. Giamati, "Application of Computer Generated Color Graphic Techniques to the Processing and Display of Three Dimensional Fluid Dynamic Data," NASA TM-82658, November 1981.

14. B. H. Anderson, C. W. Putt and C. C. Giamati, "Fluid Dynamic Data in Color and Three Dimensions," Mechanical Engineering, pp. 30-35, March 1982.

15. C. Rohrbach, F. B. Metzger, D. M. Black and R. M. Ladden, "Evaluation of Wind Tunnel Performance Testings of an Advanced $45^{\circ}$ Swept Eight-Bladed Propeller at Mach Numbers from 0.45 to 0.85 , " NASA CR-3505, March 1982 .

16. I. A. Johnsen and R. 0. Bullock, Editors, "Aerodynamic Design of Axial-Flow Compressors," NASA SP-36, 1965.

17. R. J. Jeracki, D. C. Mikkelson and B. J. Blaha, "Wind Tunnel Performance of Four Energy Efficient Propellers Designed for Mach 0.8 Cruise," NASA TM-79124, April 1979.
18. B. J. Blaha, R. Chamberlin and L. J. Bober, "Boundary Layer Thickness Effect on Boattail Drag," NASA TMX-73443, 1976.

19. O. D. Wells, M. L. Lopez, H. R. Welge, P. A. Henne and A. E. Sewell, "Wind Tunnel and Analytical Investigation of Over-the-Wing Propulsion/A ir Frame Interferences for a Short-Haul Aircraft at Mach Numbers From 0.6 to 0.78 , "NASA CR-2905, 1977.

20. J. P. Crowder, "In-Flight Propeller Flow Visualization Using Fluorescent Mini-Tufts," pp. 91-95 in "Flow Visualization and Laser Velocimetry for Wind Tunnels," NASA CP-2243, 1982.

21. G. L. Stefko, F. J. Paulovich, J. P. Greissing and E. D. Walker, "Propeller Flow Visualization Techniques," pp. 75-90 in "Flow Visualization and Laser Velocimetry for Wind Tunnels," NASA CP-2243, 1982.

22. B. J. Holmes, P. F. Coy, L. P. Yip, P. W. Brown and C. J. Obara, "Natural Laminar Flow Data From Full Scale Flight and Wind Tunnel Experiments," Eighth Annual AIAA General Aviation Technologyfest, Wichita, Kansas, November 1981.

23. R. J. Jeracki and G. A. Mitchell, "Low and High Speed Propellers for General-Aviation Performance Potential and Recent Wind Tunnel Test Results," NASA TM-81745, 1981. 


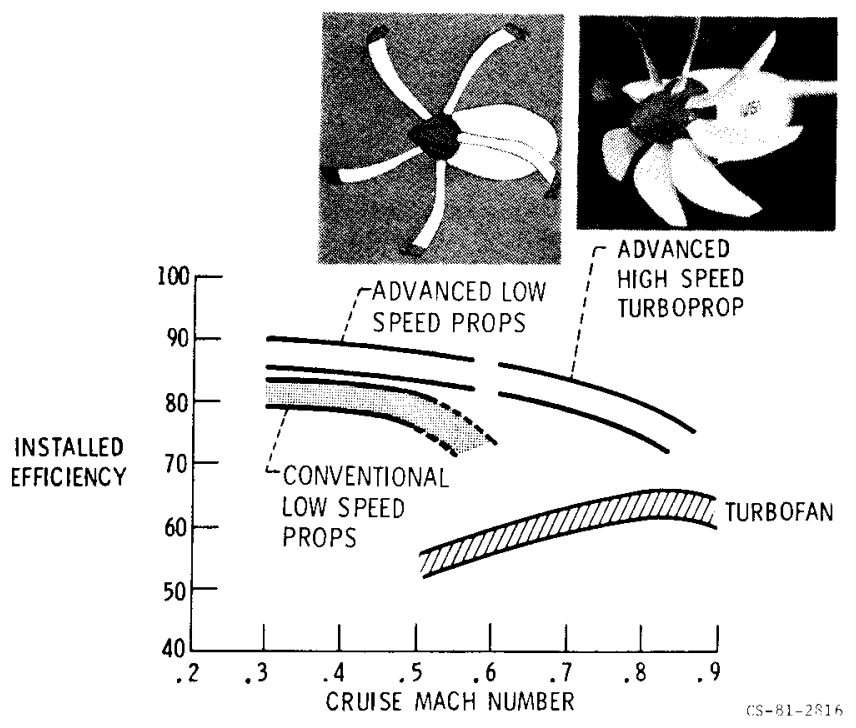

Figure 1. - Installed cruise efficiency trends of advanced turboprops compared to equivalent technology turbofans.

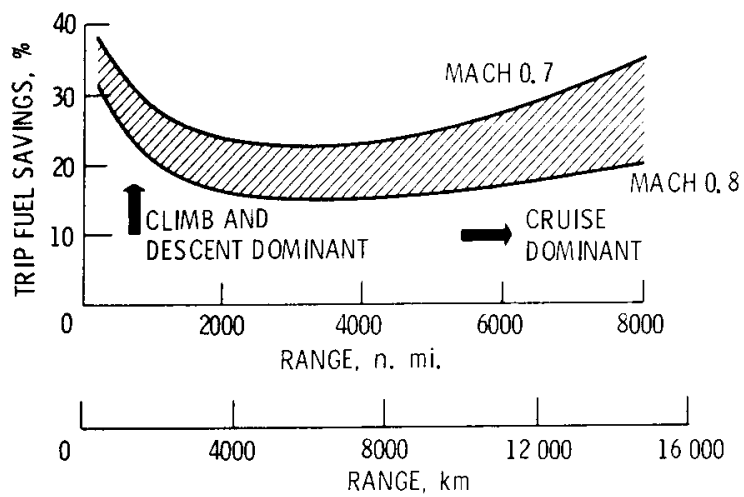

Figure 2. - Fuel savings trends of advanced tu rboprop aircraft over comparable turbofan aircraft. 


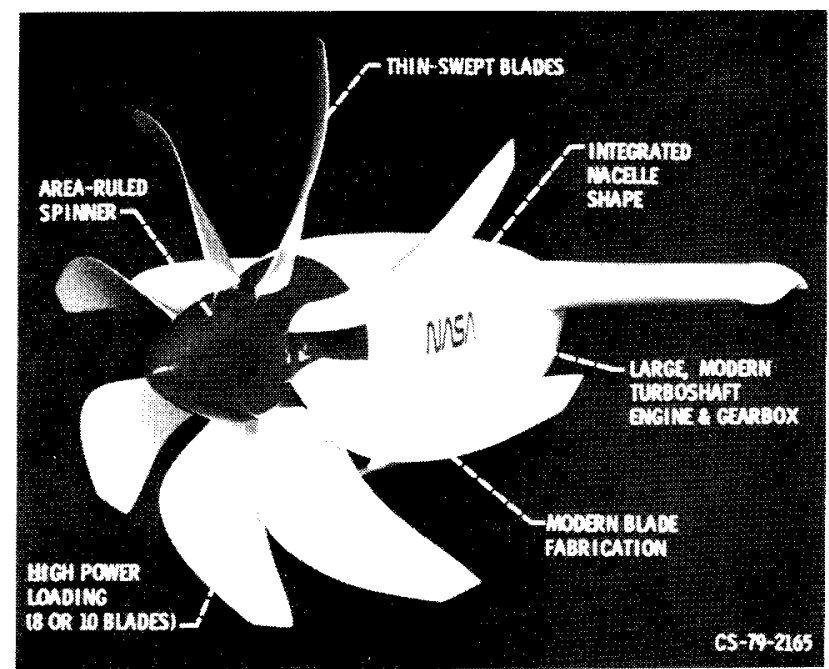

Figure 3. - Features of advanced high speed turboprop propulsion system which cause complex flow fields requiring advanced analysis.

LIFTING LINE ANALYSES

STRIP ANALYSIS - EXISTING

CURVED LIFTING LINE

PROPELLER/NACELLE

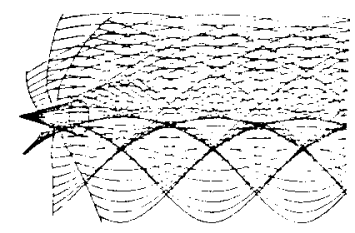

LIFTING SURFACE ANALYSES

TRANSONIC POTENTIAL

EULER EQUATIONS

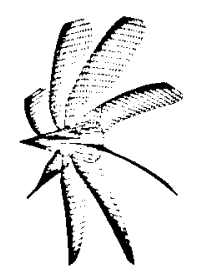

Figure 4. - Advanced analysis methods for improving propeller performance. 


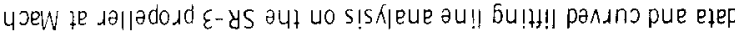

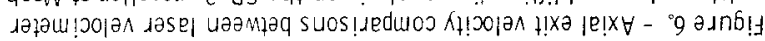
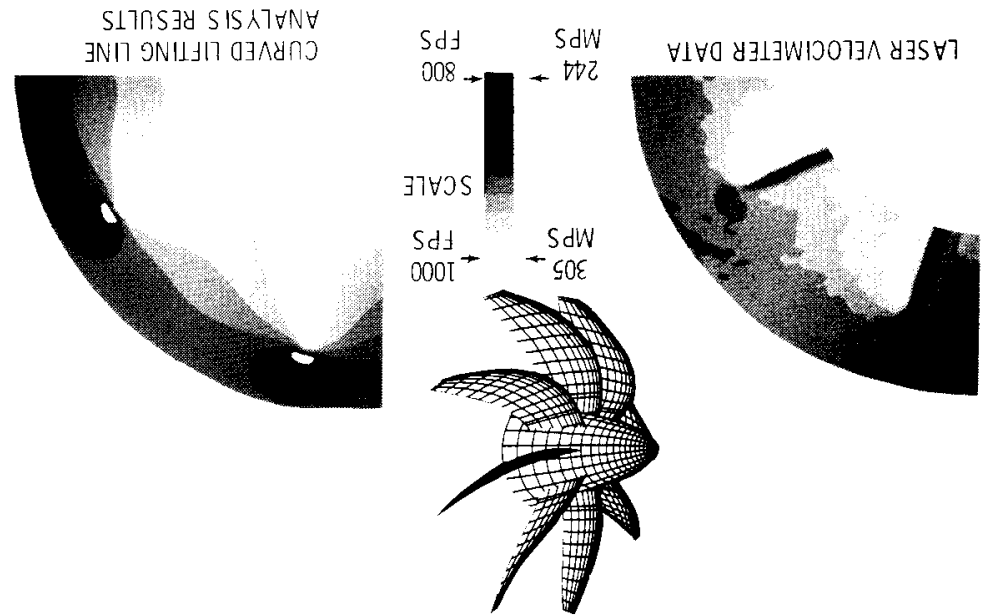

INJNOdWOJ $7 \forall I X \forall$

$\wedge 1007 \exists \wedge 11 \times \exists \varepsilon-y S$

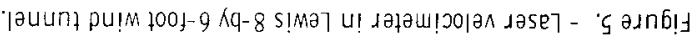

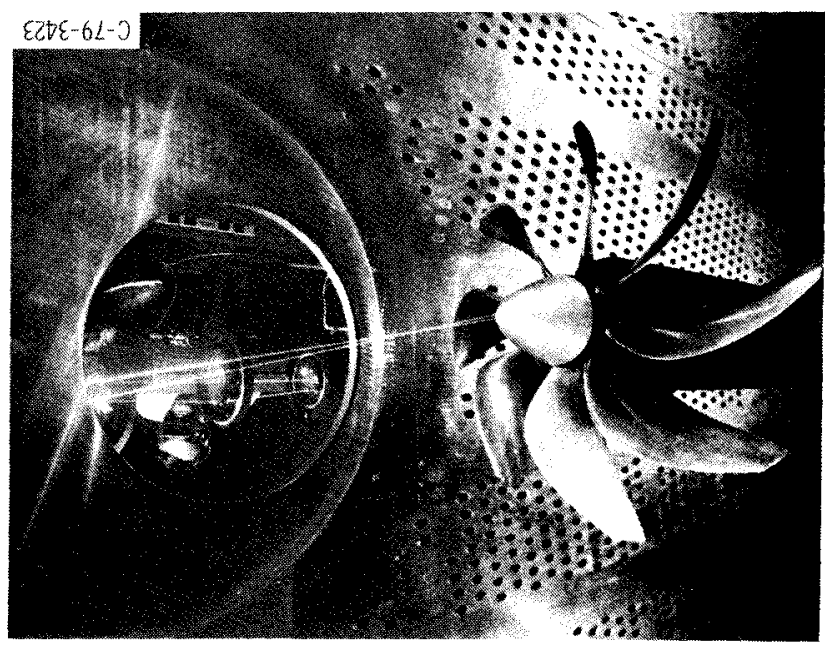




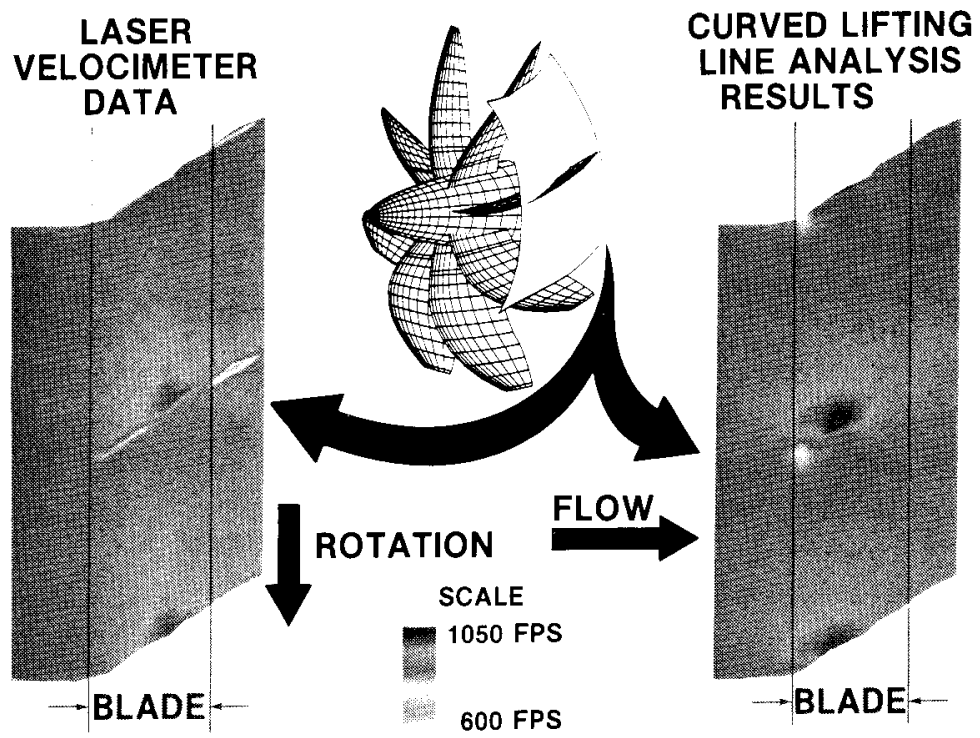

Figure 7. - Interblade resultant velocity comparisons between laser velocimeter data and the curved lifitng line analysis on the SR-3 propeller at Mach 0.80.
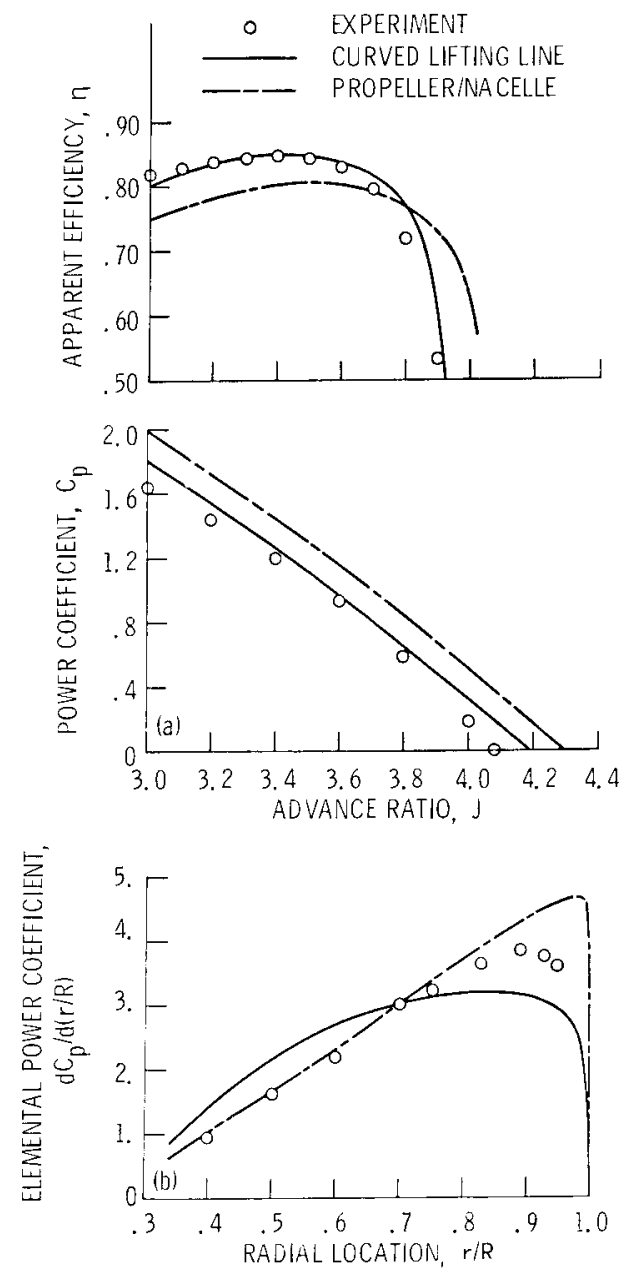

(a) Propeller performance. Blade angle at $3 / 4$ radius equals $60.4^{\circ}$.

(b) Power loading distribution, Advance ratio equals 3.06 .

Figure 8. - Con:parison of analytical and experiniental results for the $S R-3$ propeller at free stream Mach number of 0.8 . 


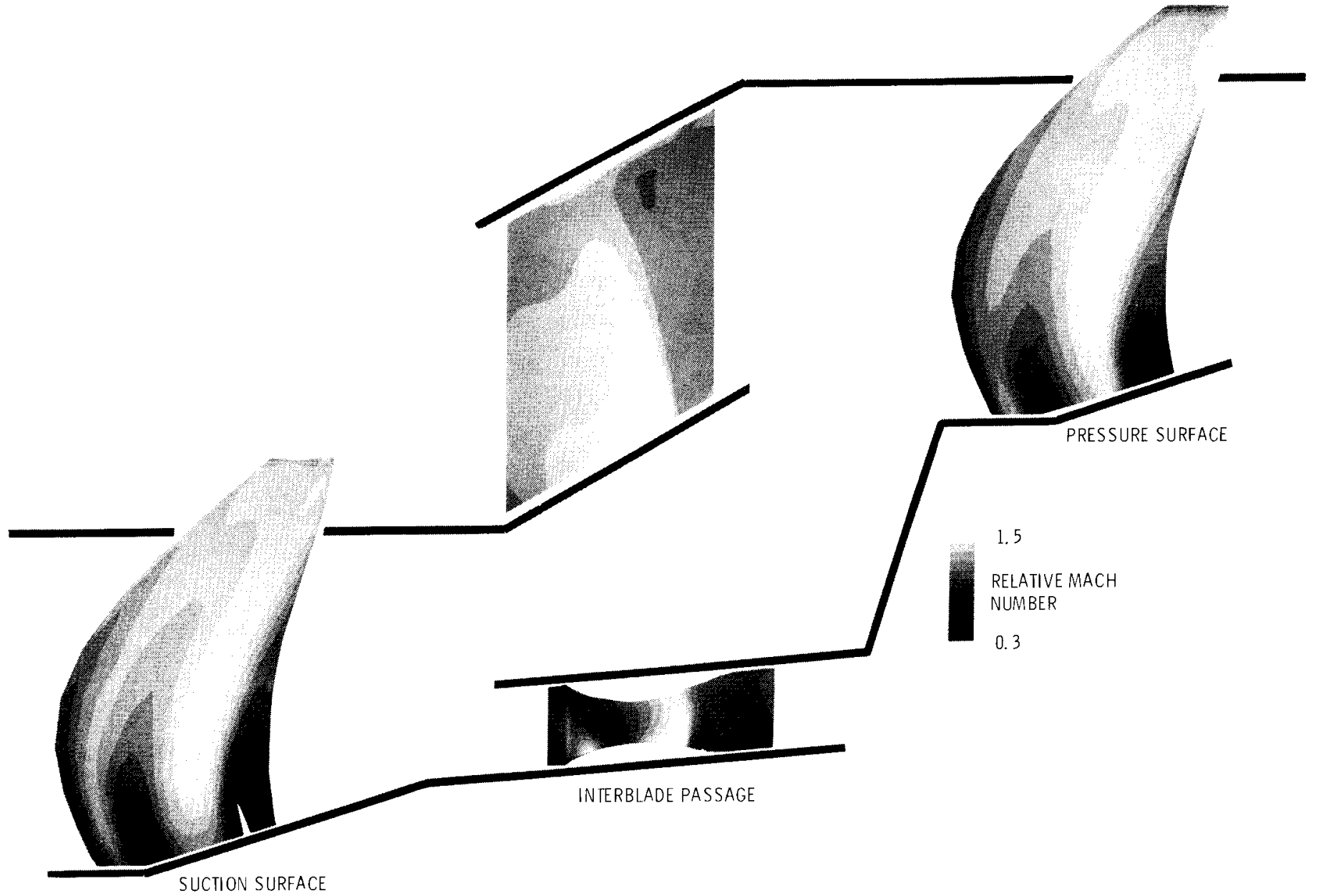

Figure 9. - Euler lifting surface analysis Mach number contours. SR-3 propeller at Mach 0.80, advance ratio 3.06, and blade angle at three-quarter radius 61.3.

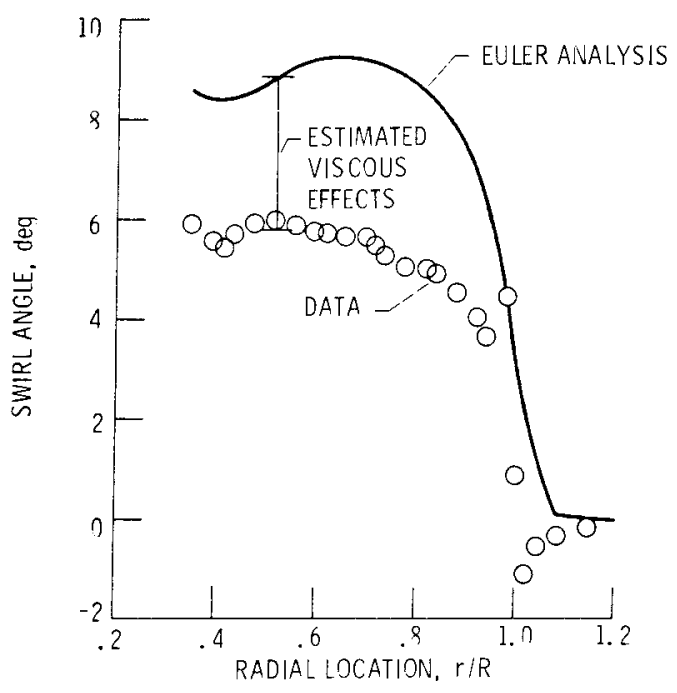

Figure 10. - Comparison of predicted and measured swirl anqle downstream of the SR-3 propeller blade. Axial location, $0.21 \mathrm{diam-}$ eters downstream of the pitch chance axis; free stream Mach number, 0.6; advance ratio, 3.06; blade angle at three-quarter radius, $60.5^{\circ}$. 


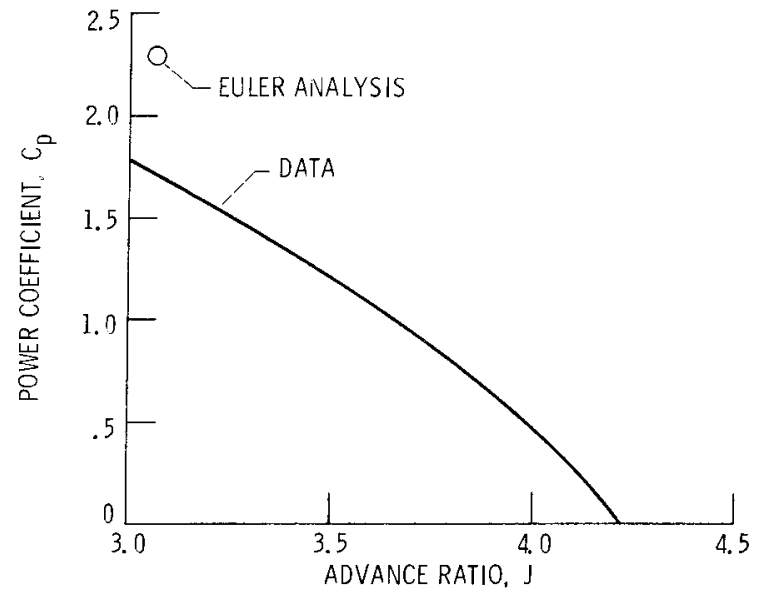

Figure 11. - Comparison of predicted and measured power coefficient for the SR-3 propeller. Free stream, Mach number, 0.6; advance ratio, 3.06; blade angle at three-quarter radius, $60.5^{\circ}$.

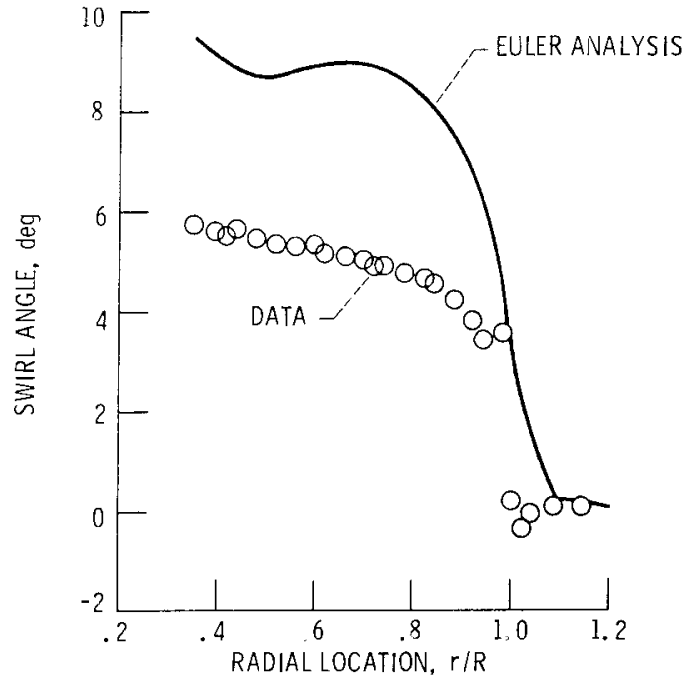

Figure 12. - Comparison of predicted and measured swirl angle downstream of the SR-3 propeller blade. Axial location, 0.21 diameters downstream of the pitch change axis. free stream Mach number, 0.8; advance ratio, 3.06; blade angle at th ree-quarter radius, $60.5^{\circ}$.

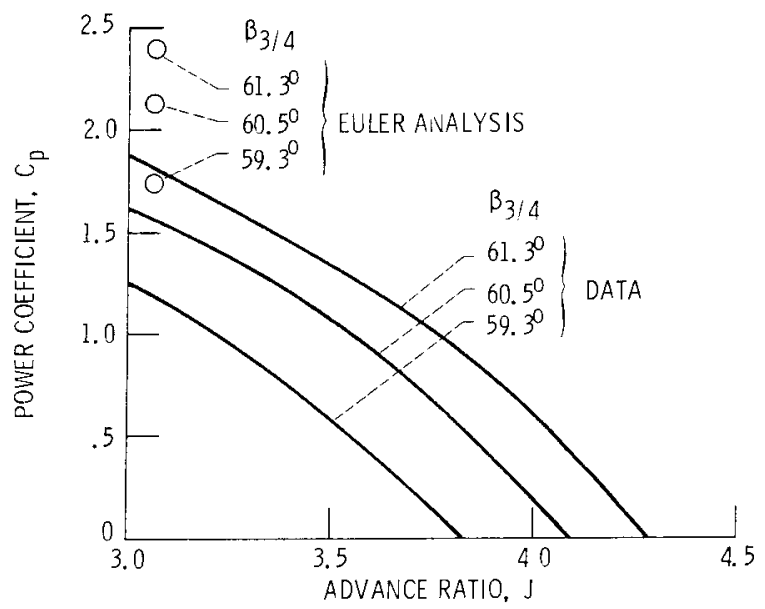

Figure 13. - Comparison of predicted and measured power coefficient for the SR-3 propeller. Free stream Mach number, 0.8; advance ratio, 3.06. 


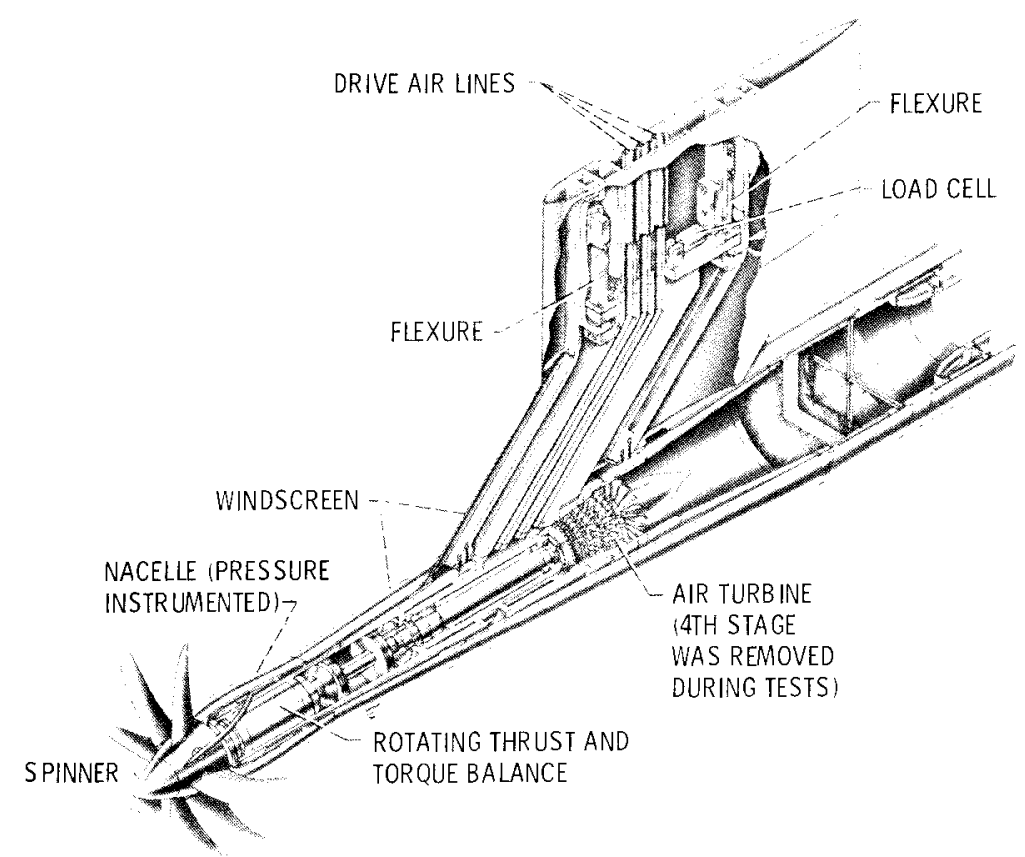

Figure 14. - Cutaway view of the Lewis 1000 horsepower $(746 \mathrm{~kW}$ ) propelter test rig.

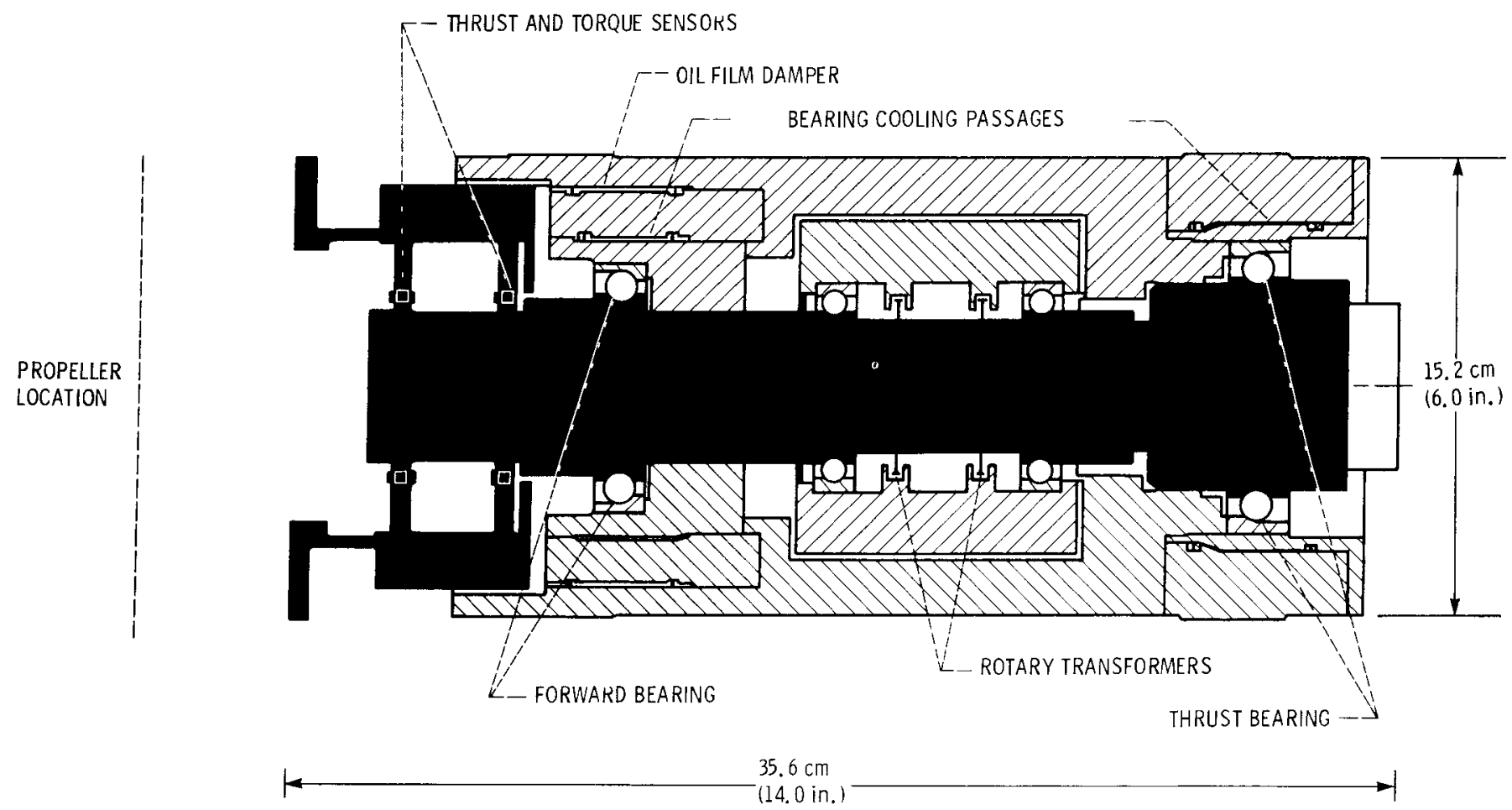

Figure 15. - New rotating thrust torque balance used for propeller testing at NASA Lewis. 


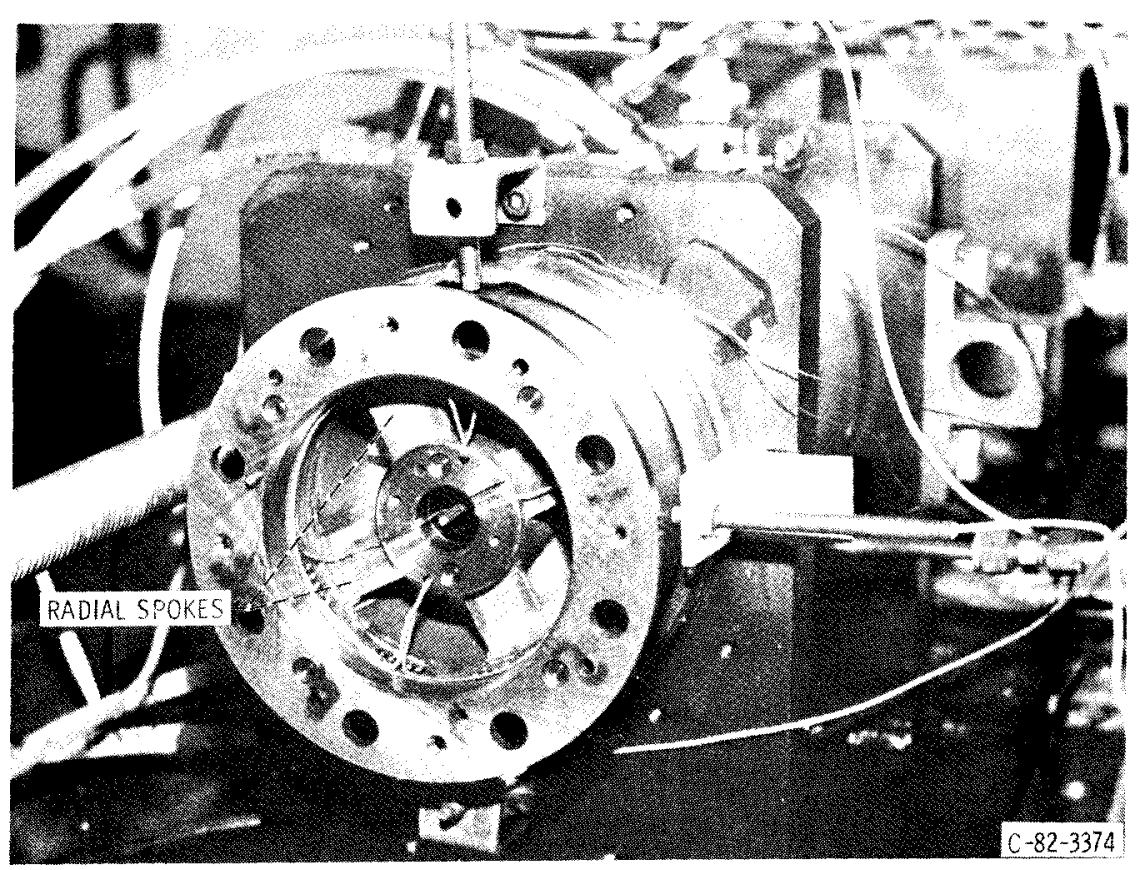

Figure 16. - Front view of the new propeller rotating balance showing the radial spokes on which the thrust and torique strain gauges are mounted.

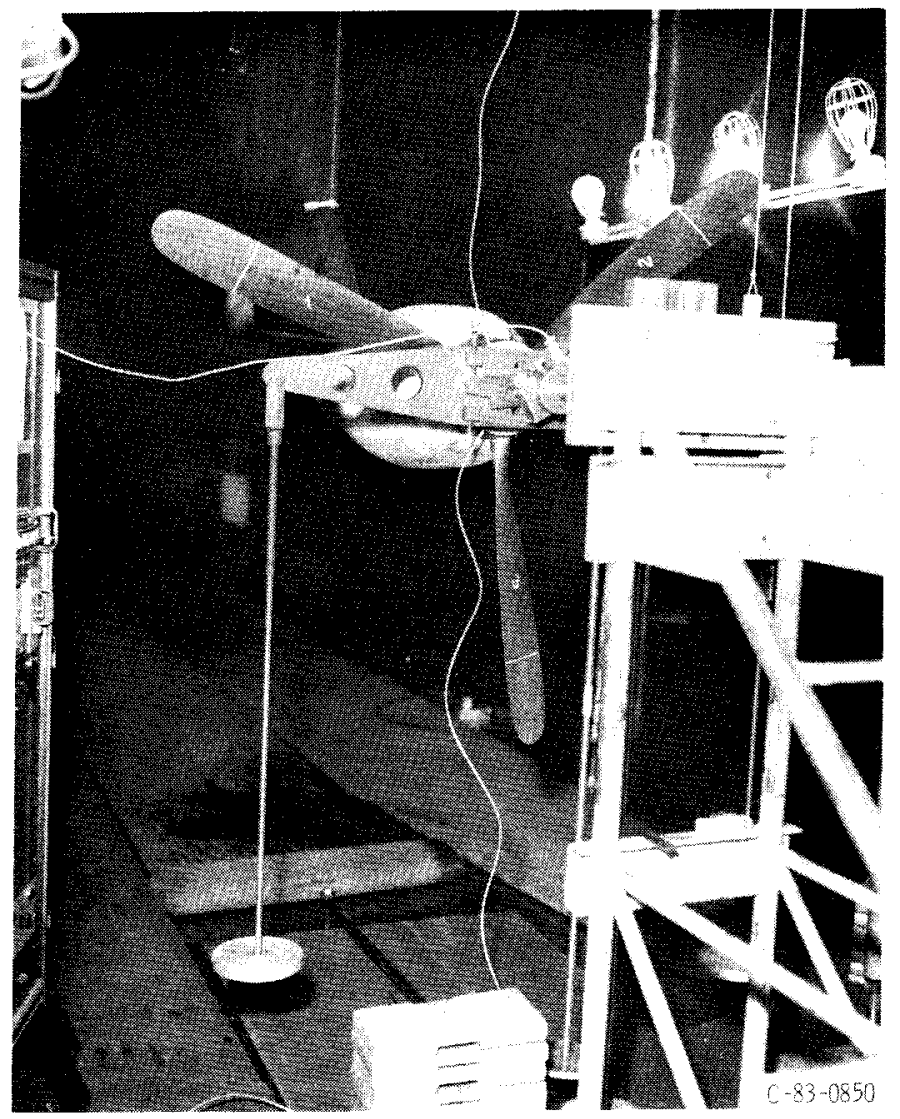

Figure 17. - Static torque calibration rig in the VASA Lewis 10-by 10 foot wind tunnel. 


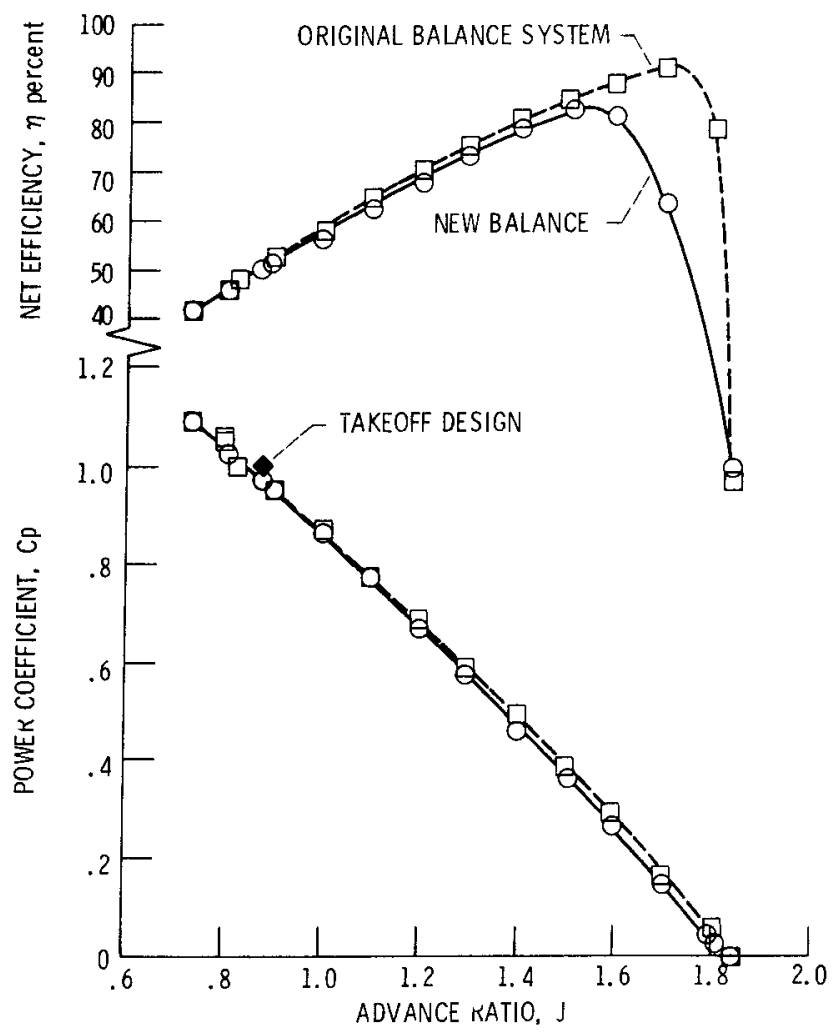

Figure 18. - Comparison of the original propeller force measurement system with the new rotating force balance for the SR-2 propeller at Mach 0.20 in the NASA Lewis $10 \times 10$ wind tunnel. 


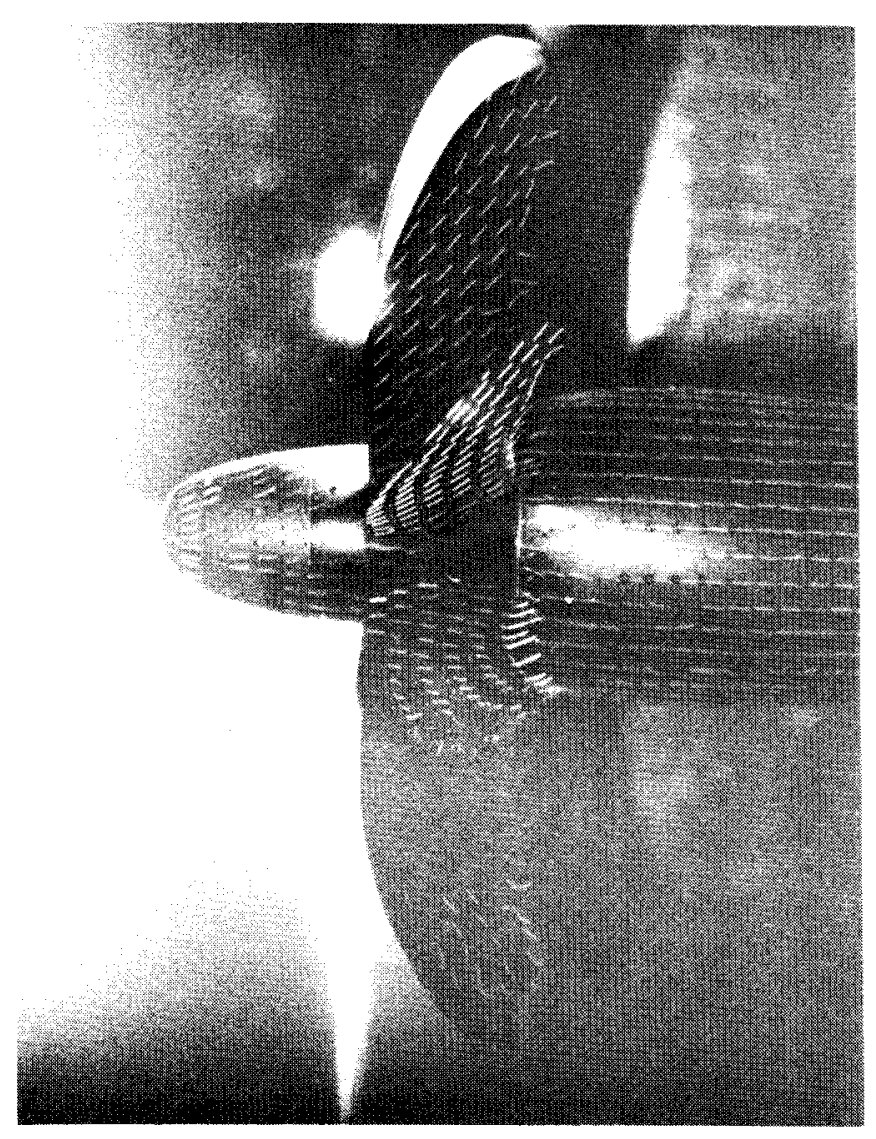

Figure 19. - Fluorescent mini-tufts on the SR-6 propeller operating at a climb condition $\left(\right.$ Mach $=0.35, R P M=5100, \beta_{3 / 4}=57^{\circ}, C_{p}=2.35$ $\mathrm{J}=2.031$

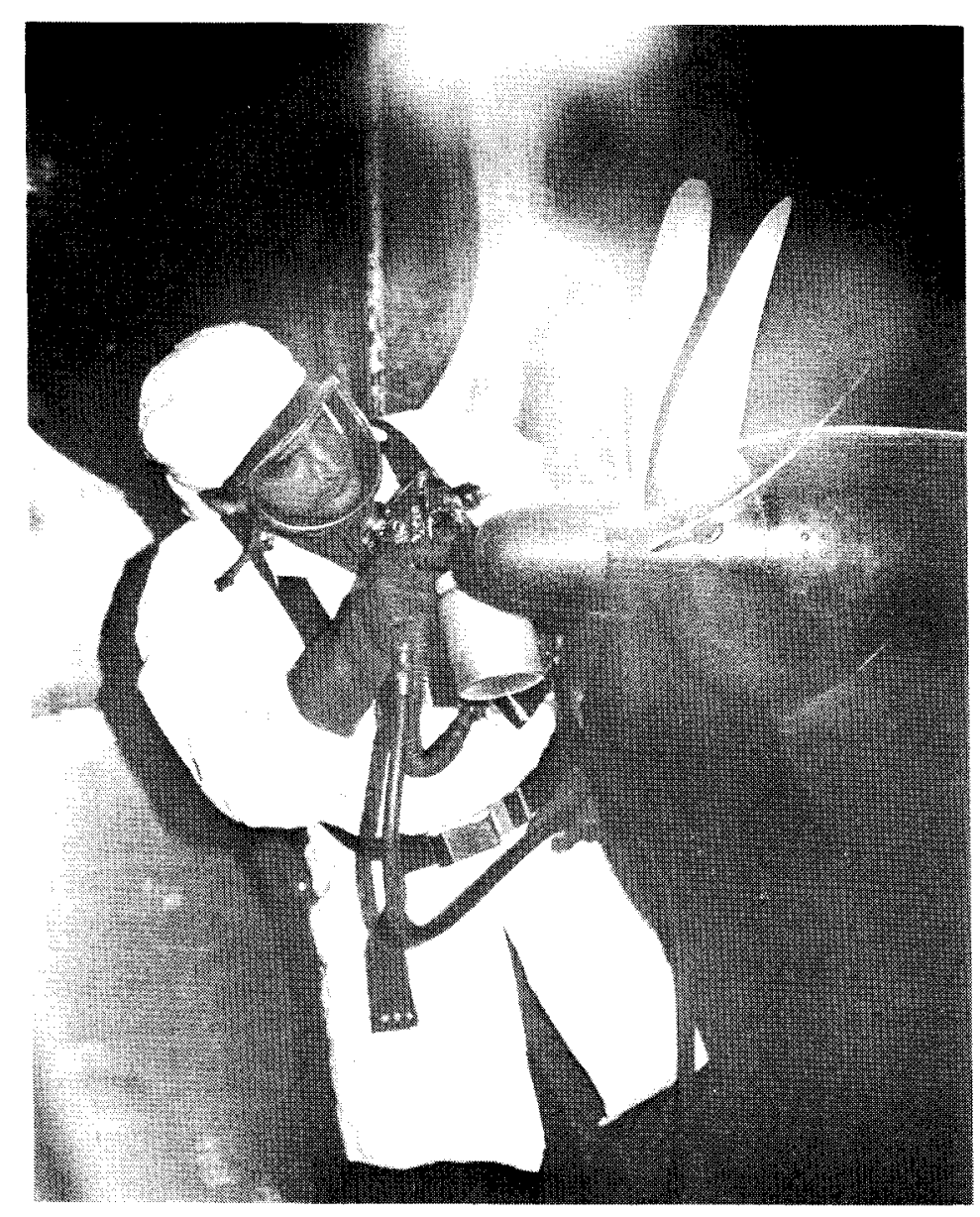

Figure 20. - Spraying the SR-6 propelter with chemicals for a sublimation flow visualization test. 


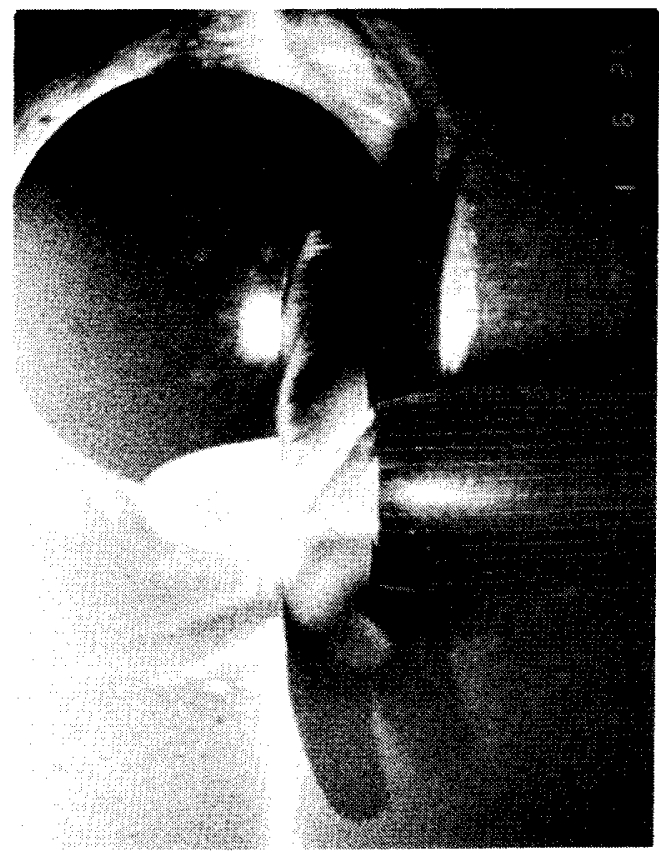

Fiqure 21. - Boundary layer transition location on the SR-6 propeller using the chemical sublimination technique. SR-6 propeller operating at a climb condition $\left(\right.$ Mach $=0.35, R P M=7300, \beta_{3 / 4}=49^{\circ}$. $C_{p}=1.59, J=1.41$ ). 


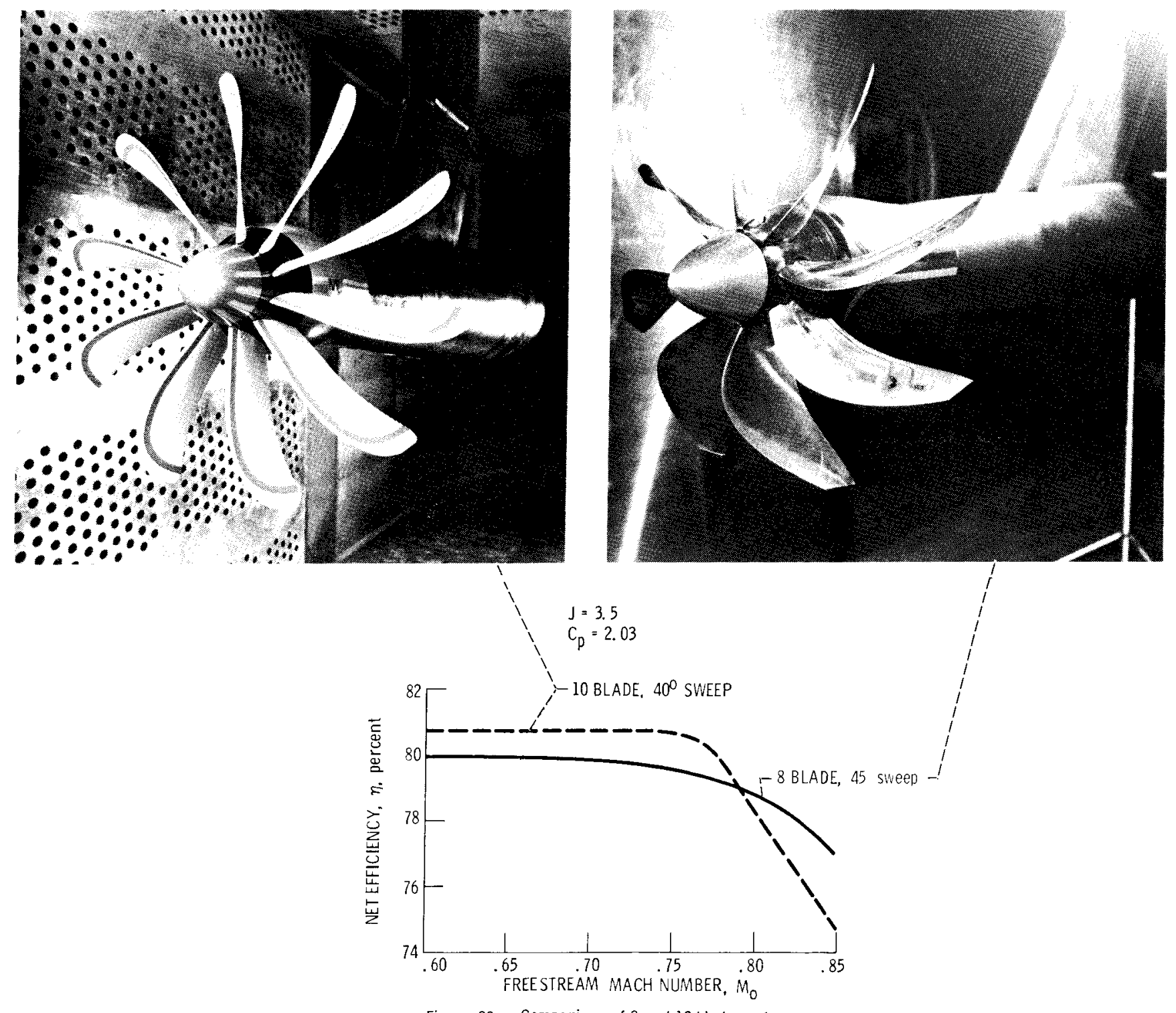

Figure 22. - Comparison of 8 and 10 blade performance. 


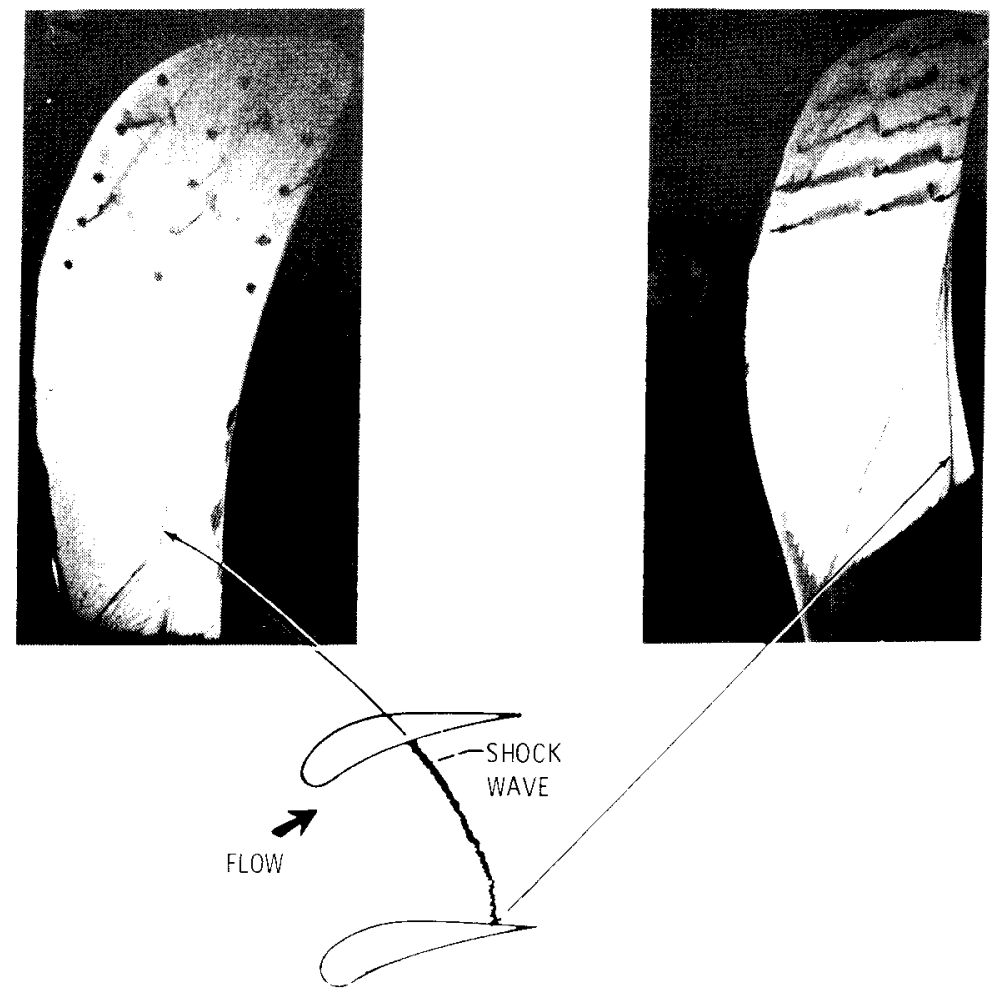

Fiqure 23. - Results from the paint flow visualization technique indicating the interblade shock structure on the 10 -bladed, $40^{\circ}$ swept propeller at Mach 0.8 . 


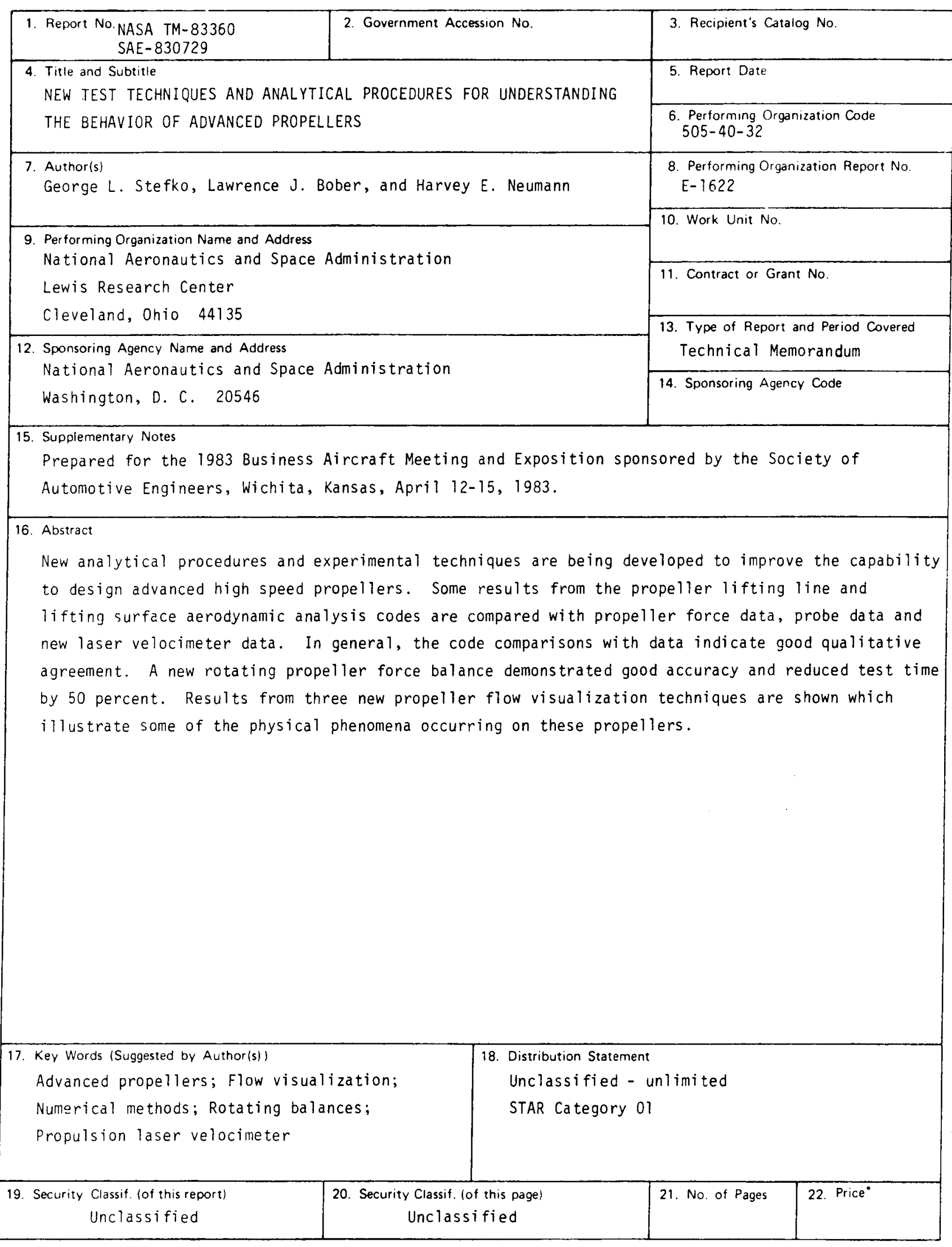

- For sale by the National Technical Inforination Service. Springfield, Vurgunla 22161 